\title{
KẾT QUẢ SỚM VÀ TRUNG HẠN TẠO HİNH VAN MỘT LÁ BẰNG MIẾNG VÁ POLYTETRAFLUOROETHYLENE (PTFE) CHO ĐƯÒ̀NG RA THẤT PHẢI TRONG PHẪU THUẠTT SỬA CHŨ̃A TRIẸT ĐỂ TỨ CHÚNG FALLOT
}

\author{
Phạm Thế Việt ${ }^{*}$, Nguyễn Văn Phan **
}

\section{TÓM TÁT}

160 bệnh nhân phẫu thuật sửa chữa triệt để tứ chứng Fallot đã được khảo sát, trong đó 109 bệnh nhân bảo tồn được vòng van ĐMP $(68,1 \%)$ cao gấp 2 lần so với số bệnh nhân được tạo hình van ĐMP với màng PTFE $0.1 \mathrm{~mm}$ (51 bệnh nhân, chiếm $31,9 \%$ ). Phẫu thuật sửa chữa tứ chứng Fallot có tạo hình van động mạch phổi một lá cho kết quả sớm và trung hạn tốt.

Tù khóa: Tứ chứng Fallot, PTFE, kết quả sớm, kết quả trung hạn

\section{EARLY AND MID-TERM RESULTS OF PTFE MONOCURP FOR PULMONARY} \section{ARTERY CORRECTION IN TETRALOGY OF FALLOT CORRECTION}

Among 160 patients of tetralogy of Fallot were performed complet correction, preservation of pulmonary valve were carried out in 109patients (68.1\%), two times higher than the group with PTFE $0.1 \mathrm{~mm}$ pulmonary monocurp ( 51 patients, $31.9 \%$ ).

Pulmonary monocusp was indicated in tetralogy of Fallot correction. The early and midterm results was satisfaisant.

\section{I. ĐẶT VẤN Đî̀}

Bảo tồn van ĐMP khi sửa chữa tứ chứng Fallot luôn được ưu tiên khi khả năng có thể bảo tồn được vì khi bảo tồn van ĐMP có thể ngăn ngừa hở van động mạch phổi, ngăn ngừa giãn thất phải diễn tiến, ngăn ngừa việc thay van động mạch phổi sau này.Ở những bệnh nhân có van ĐMP quáhẹp thì cần xẻ qua vòng van để mở rộng đường thoát thất phải và sử dụng miếng vá polytetrafluoroethylene (PTFE) $0,1 \mathrm{~mm}$. Tuy nhiên, can thiệp này có thể làm tăng nguy cơ rối loạn nhịp, suy thất phải và nguy cơ tử vong sớm sau phẫu thuật. Trong nghiên cứu này, chúng tôi đánh giá kết quả sớm và trung hạn tạo hình van một lá bằng miếng vá PTFE cho đường ra thất phải trong phẫu thuật sửa chữa tứ chứng Fallot có so sánh với kết quả của nhóm bệnh nhân được bảo tồn van ĐMP.

\section{II. ĐỐI TƯợNG VÀ PHƯƠNG PHÁP NGHIÊN CÚU}

Nghiên cứu hồi cứu với mục tiêu đánh giá kết quả sớm và trung hạn những trường hợp được phẫu thuật sửa chữa tứ chứng Fallot có tạo hình van ĐM phổi một lá có so sánh với nhóm bệnh nhân bảo tồn vòng van ĐMP.

Chúng tôi đã khảo sát được 160 hồ sơ bệnh nhân đã được phẫu thuật sửa chữa triệt để tứ chứng Fallot trong thời gian từ 1/2007 đến 1/2011, tại Bệnh viện Đại học Y Dược TP.HCM. Tất các các trường hợp này đều được tiến hành sửa chữa theo phương pháp mở nhĩ phải và động mạch phổi là phương pháp phẫu thuật không tiếp cận qua đường mở trên thất phải để sửa chữa các tổn thương tứ chứng Fallot. Tuy nhiên, với những trường hợp vòng van động mạch phổi nhỏ, chúng tôi cần xẻ qua vòng van, xẻ giới hạn $10-15 \mathrm{~mm}$

\footnotetext{
* Bệnh viện Hoàn Mỹ Sài Gòn

**Viện Tim Tp.HCM

Người chịu trách nhiệm khoa họ: TS Nguyễn Văn Phan

Ngày nhận bài: 20/03/2015 - Ngày Cho Phép Đăng: 27/03/2015

Phản Biện Khoa hoc: PGS.TS. Đặng Ngoc Hùng

PGS.TS. Lê Ngọc Thành
} 
vào thất phải để mở rộng đường thoát thất phải và miếng vá PTFE $0,1 \mathrm{~mm}$ được dùng để tạo hình van động mạch phổi một lá. Trong 160bệnh nhân, có 109 bệnh nhân được bảo tồn vòng van ĐMP và có 51 bệnh nhân cần xẻ qua vòng van động mạch phổi tạo hình van một lá.

Số liệu theo dõi bệnh nhân sau phẫu thuật được thu thập với thời gian tối thiểu 1 năm. Đánh giá kết quả phẫu thuật sửa chữa triệt để tứ chứng Fallot có tạo hình van động mạch phổi một lá (tốt/ không tốt) dựa vào chất lượng sống (đánh giá mức độ mệt trên lâm sàng), siêu âm tim đánh giá (chức năng hai thất, thông liên thất tồn lưu, hở van động mạch phổi, chênh áp qua đường thoát thất phải, hở van ba lá), tử vong trong thời gian tối thiểu 1 năm sau khi được phẫu thuật.

\section{KẾT QUẢ}

\section{1. Đặc điểm bệnh nhân trước mổ}

Bảng 1: Đặc điểm bệnh nhân trước mổ phân bố theo chỉ định can thiệp (bảo tồn van ĐMP hoặc PTFE)

\begin{tabular}{|c|c|c|c|c|c|}
\hline \multirow{2}{*}{ Đặc điểm } & \multicolumn{2}{|c|}{ PTFE $(n=51)$} & \multicolumn{2}{|c|}{$\begin{array}{c}\text { Tồn lưu van ĐMP } \\
(\mathrm{n}=109)\end{array}$} & \multirow[t]{2}{*}{ Giá trị $p$} \\
\hline & $\begin{array}{l}\text { Tần } \\
\text { suất }\end{array}$ & Tỉ lệ (\%) & Tần suất & Tỉ lệ (\%) & \\
\hline \multicolumn{6}{|l|}{ Giới tính } \\
\hline Nam & 24 & 47,06 & 55 & 50,46 & \multirow[t]{2}{*}{0,69} \\
\hline Nữ & 27 & 52,94 & 54 & 49,54 & \\
\hline $\begin{array}{c}\text { Tuổi } \\
\text { (trung bình, min-max) }\end{array}$ & \multicolumn{2}{|c|}{$\begin{array}{c}10,03 \pm 5,54 \\
(2-36)\end{array}$} & \multicolumn{2}{|c|}{$\begin{array}{c}9,85 \pm 5,76 \\
(2-36)\end{array}$} & 0,55 \\
\hline $\begin{array}{c}\text { Cân nặng } \\
\text { (trung bình, min-max) } \\
\text { trung vị }\end{array}$ & \multicolumn{2}{|c|}{$\begin{array}{c}22,99 \pm 10,4 \\
(10.4-50) \\
20\end{array}$} & \multicolumn{2}{|c|}{$\begin{array}{c}21,98 \pm 9,69 \\
(10-47) \\
019,5\end{array}$} & 0,55 \\
\hline $\mathrm{BSA}$ & \multicolumn{2}{|c|}{$\begin{array}{c}0,89 \pm 0,27 \\
(0,5-1,5)\end{array}$} & \multicolumn{2}{|c|}{$\begin{array}{l}0,87 \pm 0,27 \\
(0,45-1,48)\end{array}$} & 0,62 \\
\hline \multicolumn{6}{|l|}{ Trước phẫu thuật } \\
\hline $\begin{array}{l}\text { Chênh áp qua đường thoát thất } \\
\text { phải }(\mathrm{mm} \mathrm{Hg})\end{array}$ & \multicolumn{2}{|c|}{$\begin{array}{c}75,55 \pm 12,82 \\
(50-108) \\
\end{array}$} & \multicolumn{2}{|c|}{$\begin{array}{c}73.33 \pm 12.3 \\
(50-101)\end{array}$} & 0,30 \\
\hline ĐK Thất trái tâm trương (mm) & \multicolumn{2}{|c|}{$\begin{array}{c}27,92 \pm 5,42 \\
(18-41,9)\end{array}$} & \multicolumn{2}{|c|}{$\begin{array}{c}29,41 \pm 5,98 \\
(17-44,8)\end{array}$} & 0,111 \\
\hline Vòng van ĐMP (mm) & \multicolumn{2}{|c|}{$\begin{array}{l}11,93 \pm 3,09 \\
(7-21)\end{array}$} & \multicolumn{2}{|c|}{$\begin{array}{c}13,36 \pm 3,13 \\
(7-24,5)\end{array}$} & 0,008 \\
\hline
\end{tabular}

Kết quả khảo sát các đặc điểm giữa 2 nhóm van ĐMP là 13,36 mm rộng hơn so với nhóm bệnh nhân (bảng 1), chúng tôi ghi nhận các đặc điểm phân bố đồng đều và không có khác biệt về thống kê. Tuy nhiên, có khác biệt có ý nghĩa thống kê về đường kính vòng van ĐMP ở nhóm tồn lưu PTFE 11,93 mm ( $\mathrm{p}=0,008)$.

Để đảm bảo sự đồng nhất và hạn chế sự chênh lệch khi tiến hành so sánh giữa 2 nhóm, chúng tôi tiến hành phân nhóm bệnh nhân theo 
chỉ số $Z$ vòng van ĐMP với ngưỡng là -3 , với nhóm $A(Z<-3)$ và nhóm $B(Z \geq-3)$. Kết quả so sánh đường kính vòng van ĐMP cho thấy không có sự khác biệt ở mỗi phân nhóm (bảng 2), do đó chúng tôi tiến hành khảo sát so sánh các kết quả sớm và trung hạn của kỹ thuật tạo hình van một lá bằng $\mathrm{PTFE}$ với kết quả của bảo tồn van ĐMP theo phân nhóm $A$ và $B$ này.

Bảng 2: Đường kính vòng van ĐMP ở hai phân nhóm $A$ và $B$

\begin{tabular}{|c|c|c|c|c|c|}
\hline \multirow[b]{2}{*}{ Đặc điểm } & \multicolumn{2}{|c|}{ PTFE } & \multicolumn{2}{|c|}{ Bảo tồn van ĐMP } & \multirow[b]{2}{*}{ Giá trị p } \\
\hline & Tần suất & $\begin{array}{l}\text { Tỉ lệ } \\
\text { (\%) }\end{array}$ & Tần suất & $\begin{array}{l}\text { Tỉ lệ } \\
(\%)\end{array}$ & \\
\hline Nhóm A (Z<-3) & \multicolumn{2}{|c|}{$\mathrm{n}=22$} & \multicolumn{2}{|c|}{$n=13$} & \\
\hline Vòng van ĐMP (mm) & \multicolumn{2}{|c|}{$10,4 \pm 0,52$} & \multicolumn{2}{|c|}{$9,93 \pm 0,80$} & 0,63 \\
\hline Nhóm $B(Z \geq-3)$ & \multicolumn{2}{|c|}{$\mathrm{n}=\mathbf{2 9}$} & \multicolumn{2}{|c|}{$n=96$} & \\
\hline Vòng van ĐMP (mm) & \multicolumn{2}{|c|}{$13,08 \pm 0,56$} & \multicolumn{2}{|c|}{$13,82 \pm 0,29$} & 0,25 \\
\hline
\end{tabular}

\section{2. Đánh giá kết quả sớm}

Các chỉ số liên quan đến quá trình phẫu thuật và kết quả sớm sau phẫu thuật ghi nhận không có sự khác biệt giữa 2 nhóm bệnh nhân PTFE và Bảo tồn van ĐMP $(\mathrm{p}>0,05)$. Tuy nhiên, ở bệnh nhân có chỉ số $\mathrm{Z}<-3$ thì ở nhóm
PTFE cải thiện chỉ số chênh áp qua đường thoát thất phải tốt hơn so với nhóm Bảo tồn van ĐMP $(\mathrm{p}=0,005)$, điều này cho thấy ở những bệnh nhân nặng $(Z<-3)$ cần dùng PTFE. (Xem kết quả ở bảng 3).

Bảng 3: Kết quả sớm giũa PTFE so với Bảo tồn van ĐMP theo phân nhóm A và B

\begin{tabular}{|c|c|c|c|c|c|}
\hline \multirow{2}{*}{ Đặc điểm } & \multicolumn{2}{|c|}{ PTFE } & \multicolumn{2}{|c|}{$\begin{array}{c}\text { Bảo tồn van } \\
\text { ĐMP }\end{array}$} & \multirow{2}{*}{ Giá trị p } \\
\hline & Tần suất & $\begin{array}{l}\text { Tỉ lệ } \\
(\%)\end{array}$ & $\begin{array}{l}\text { Tần } \\
\text { suất }\end{array}$ & $\begin{array}{l}\text { Tỉ lệ } \\
(\%)\end{array}$ & \\
\hline Nhóm A $(Z<-3)$ & \multicolumn{2}{|c|}{$\mathrm{n}=\mathbf{2 2}$} & \multicolumn{2}{|c|}{$\mathrm{n}=\mathbf{1 3}$} & \\
\hline Thời gian nằm hồi sức & \multicolumn{2}{|c|}{$4,45 \pm 0,52$} & \multicolumn{2}{|c|}{$5,92 \pm 1,39$} & 0,25 \\
\hline Thời gian thở máy & \multicolumn{2}{|c|}{$28,09 \pm 5,38$} & \multicolumn{2}{|c|}{$21,7 \pm 4,34$} & 0,42 \\
\hline Chênh áp qua đường thoát thất phải & \multicolumn{2}{|c|}{$20,63 \pm 2,04$} & \multicolumn{2}{|c|}{$32 \pm 3,46$} & 0,005 \\
\hline \multicolumn{6}{|l|}{ Hở ĐMP } \\
\hline Có & \multicolumn{2}{|c|}{$13(59,09 \%)$} & \multicolumn{2}{|c|}{$5(38,46 \%)$} & \multirow[t]{2}{*}{0,24} \\
\hline Không & \multicolumn{2}{|c|}{$9(40,91 \%)$} & \multicolumn{2}{|c|}{$8(61,54 \%)$} & \\
\hline Nhóm $B(Z \geq-3)$ & \multicolumn{2}{|c|}{$\mathrm{n}=\mathbf{2 9}$} & \multicolumn{2}{|c|}{$n=96$} & \\
\hline
\end{tabular}




\begin{tabular}{|c|c|c|c|c|c|}
\hline \multirow{2}{*}{ Đặc điểm } & \multicolumn{2}{|c|}{ PTFE } & \multicolumn{2}{|c|}{$\begin{array}{c}\text { Bảo tồn van } \\
\text { ĐMP }\end{array}$} & \multirow{2}{*}{ Giá trị p } \\
\hline & Tần suất & $\begin{array}{l}\text { Tỉ lệ } \\
(\%)\end{array}$ & $\begin{array}{l}\text { Tần } \\
\text { suất }\end{array}$ & $\begin{array}{l}\text { Tỉ lệ } \\
(\%)\end{array}$ & \\
\hline Thời gian nằm hồi sức & \multicolumn{2}{|c|}{$5,13 \pm 1,34$} & \multicolumn{2}{|c|}{$3,54 \pm 0,18$} & 0,05 \\
\hline Thời gian thở máy & \multicolumn{2}{|c|}{$25,84 \pm 4,95$} & \multicolumn{2}{|c|}{$17,03 \pm 1,96$} & 0,05 \\
\hline Chênh áp qua đường thoát thất phải & \multicolumn{2}{|c|}{$26,13 \pm 2,18$} & \multicolumn{2}{|c|}{$22,54 \pm 1,14$} & 0,14 \\
\hline \multicolumn{6}{|l|}{ Hở ĐMP } \\
\hline Có & \multicolumn{2}{|c|}{$20(68,97 \%)$} & \multicolumn{2}{|c|}{$59(61,46 \%)$} & \multirow[t]{2}{*}{0,46} \\
\hline Không & \multicolumn{2}{|c|}{$9(31,03 \%)$} & \multicolumn{2}{|c|}{$37(38,54 \%)$} & \\
\hline
\end{tabular}

\section{3. Đánh giá kết quả trung hạn}

Các chỉ số liên quan đến quá trình theo dõi theo thời gian (trung hạn) ghi nhận không có sự khác biệt giữa 2 nhóm bệnh nhân PTFE và Bảo tồn van ĐMP (p>0,05). Tuy nhiên, ở bệnh nhân có chỉ số Z<-3 thì ở nhóm PTFE đã duy trì tốt chỉ số chênh áp qua đường thoát thất phải tốt hơn so với nhóm Bảo tồn van ĐMP ( $\mathrm{p}=0,009)$, theo như kết quả của nghiên cứu này cho thấy kỹ thuật PTFE duy trì hiệu quả khi xét đến thời điểm trung hạn. (Xem kết quả ở bảng 4).

Bảng 4: Kết quả trung hạn giũua PTFE so với Bảo tồn van ĐMP theo phân nhóm A và $B$

\begin{tabular}{|c|c|c|c|c|c|}
\hline \multirow{2}{*}{ Đặc điểm } & \multicolumn{2}{|c|}{ PTFE } & \multicolumn{2}{|c|}{$\begin{array}{c}\text { Bảo tồn van } \\
\text { ĐMP }\end{array}$} & \multirow{2}{*}{ Giá trị p } \\
\hline & Tần suất & $\begin{array}{l}\text { Tỉ lệ } \\
\text { (\%) }\end{array}$ & $\begin{array}{l}\text { Tần } \\
\text { suất }\end{array}$ & $\begin{array}{l}\text { Tỉ lệ } \\
\text { (\%) }\end{array}$ & \\
\hline Nhóm A $(Z<-3)$ & \multicolumn{2}{|c|}{$\mathrm{n}=\mathbf{2 2}$} & \multicolumn{2}{|c|}{$n=13$} & \\
\hline Tử vong & \multicolumn{2}{|c|}{0} & \multicolumn{2}{|c|}{0} & \\
\hline Phẫu thuật lại & \multicolumn{2}{|c|}{0} & \multicolumn{2}{|c|}{0} & \\
\hline Chênh áp qua đường thoát thất phải & \multicolumn{2}{|c|}{$18,25 \pm 1,79$} & \multicolumn{2}{|c|}{$27,7 \pm 3,26$} & 0,009 \\
\hline \multicolumn{6}{|l|}{ Hở ĐMP } \\
\hline Có & \multicolumn{2}{|c|}{$13(59,09 \%)$} & \multicolumn{2}{|c|}{$5(38,46 \%)$} & \multirow[t]{2}{*}{0,24} \\
\hline Không & \multicolumn{2}{|c|}{$9(40,91 \%)$} & \multicolumn{2}{|c|}{$8(61,54 \%)$} & \\
\hline \multicolumn{6}{|l|}{ Hở van 3 lá } \\
\hline Có & \multicolumn{2}{|c|}{$25 \%$} & \multicolumn{2}{|c|}{$30 \%$} & \multirow[t]{2}{*}{0,95} \\
\hline Không & \multicolumn{2}{|c|}{$75 \%$} & \multicolumn{2}{|c|}{$70 \%$} & \\
\hline Nhóm $B(Z \geq-3)$ & \multicolumn{2}{|c|}{$n=29$} & \multicolumn{2}{|c|}{$n=96$} & \\
\hline
\end{tabular}




\begin{tabular}{|c|c|c|c|c|c|}
\hline \multirow{2}{*}{ Đặc điểm } & \multicolumn{2}{|c|}{ PTFE } & \multicolumn{2}{|c|}{$\begin{array}{l}\text { Bảo tồn van } \\
\text { ĐMP }\end{array}$} & \multirow{2}{*}{ Giá trị $\mathrm{p}$} \\
\hline & Tần suất & $\begin{array}{l}\text { Tỉ lệ } \\
\text { (\%) }\end{array}$ & $\begin{array}{l}\text { Tần } \\
\text { suất }\end{array}$ & $\begin{array}{l}\text { Tỉ lệ } \\
\text { (\%) }\end{array}$ & \\
\hline Tử vong & \multicolumn{2}{|c|}{0} & \multicolumn{2}{|c|}{0} & \\
\hline Phẫu thuật lại & \multicolumn{2}{|c|}{0} & \multicolumn{2}{|c|}{0} & \\
\hline Chênh áp qua đường thoát thất phải & \multicolumn{2}{|c|}{$21,5 \pm 2,20$} & \multicolumn{2}{|c|}{$20,26 \pm 0,97$} & 0,56 \\
\hline \multicolumn{6}{|l|}{ Hở ĐMP } \\
\hline Có & \multicolumn{2}{|c|}{$20(68,97 \%)$} & \multicolumn{2}{|c|}{$59(61,46 \%)$} & \multirow[t]{2}{*}{0,46} \\
\hline Không & \multicolumn{2}{|c|}{$9(31,03 \%)$} & \multicolumn{2}{|c|}{$37(38,54 \%)$} & \\
\hline \multicolumn{6}{|l|}{ Hở van 3 lá } \\
\hline Có & \multicolumn{2}{|c|}{$20 \%$} & \multicolumn{2}{|c|}{$25 \%$} & \multirow[t]{2}{*}{0,92} \\
\hline Không & \multicolumn{2}{|c|}{$80 \%$} & \multicolumn{2}{|c|}{$75 \%$} & \\
\hline
\end{tabular}

\section{BÀN LUẬN}

Kết quả so sánh các đặc điểm dân số nghiên cứu khi theo phân nhóm và theo kỹ thuật can thiệp ghi nhận các đặc điểm phân bố đồng đều điều này thuận lợi cho việc so sánh kết quả phẫu thuật khi giữa các nhóm không có những yếu tố chênh lệch gây nhiễu. Trong dân số nghiên cứu, chúng tôi có 29 bệnh nhân có chỉ số $Z>-3$ nhưng vẫn được chỉ định can thiệp bằng PTFE vì có những triệu chứng nặng khác như hẹp dưới van ĐMP, vị trí thông liên thất và hẹp nhánh.

Tình trạng trong và sau phẫu thuật như thời gian thở máy, thời gian nằm hồi sức trong nghiên cứu này, chúng tôi ghi nhận giữa 2 nhóm không có khác biệt. Tuy nhiên,kết quả này trái ngược với các công trình trên thế giới, chẳng hạn, tác giả Sasson L. (2013) ghi nhận ở nhóm Bảo tồn van ĐMP lại có thời gian thở máy, thời gian nằm hồi sức lâu hơn nhóm PTFE $(\mathrm{p}<0,01)$; tác giả Mark W (2002) ghi nhận thời gian nằm hồi sức ở nhóm PTFE ngắn hơn nhóm Bảo tồn van ĐMP $(3,6$ ngày so với 5,8 ngày, $\mathrm{p}<0,05$ ). Điều này có thể lý giải khả năng liên quan đến các kỹ thuật chăm sóc hậu phẫu ở những đơn vị khác nhau thì có thể ảnh hưởng đến kết quả ghi nhận, ở các nước phát triển thì các điều kiện chăm sóc có thể tốt hơn so với tình hình tại Việt Nam nói chung.

Kết quả sớm và trung hạn, trong nghiên cứu này, chúng tôi ghi nhận hiệu quả của PTFE tốt khi có thể cải thiện và duy trì chỉ số chênh áp đường ra thất phải tốt hơn so với nhóm Bảo tồn van ĐMP. Ngoài ra, các đặc điểm lâm sàng khác đều tốt và không có sự khác biệt giữa 2 nhóm bệnh nhân (PTFE so với Bảo tồn van ĐMP). Kết quả này tương tự với các công trình nghiên cứu đánh giá hiệu quả của PTFE trong và ngoài nước.Tác giả N.B.Khoa (2011) ghi nhận độ chênh áp tối đa qua van động mạch phổi trước mổ là 77,72 $\pm 10,8$ $\mathrm{mmHg}$, giảm còn $21.04 \pm 12.53 \mathrm{mmHg}$ khi xuất viện. Khi tái khám sau thời gian tối thiểu 1 năm, độ chênh áp này giữ ở mức $13,91 \pm 6,38 \mathrm{mmHg}$ ở nhóm 32 bệnh nhân được theo dõi và không có bệnh nhân nào hở phổi nặng. Tác giả Mark $\mathrm{W}$ (2002) ghi nhận không có sự khác biệt về chênh áp 
qua đường thoát thất phải, mức độ hở van 3 lá, hở van ĐMP giữa nhóm bệnh nhân PTFE so với với Bảo tồn van ĐMP $(\mathrm{p}>0,05)$.

\section{KẾT LUẬN}

Việc tạo hình van một lá bằng miếng vá PTFE cho đường ra thất phải trong phẫu thuật sửa chữa tứ chứng Fallotkết quả sớm và trung hạn giảm hở van ĐMP và duy trì chênh áp đường ra thất phải tốt. Kỹ thuật PTFE dễ thực hiện và an toàn, bệnh nhân được dùng PTFE có thời gian hồi sức và thở máy tương tự với bệnh nhân được bảo tồn van ĐMP.

\section{TÀI LIỆU THAM KHẢO}

1. Nguyễn Sinh Hiền, (2011), "Nghiên cứu ứng dụng kỹ thuật không mở thất phải trong điều trị phẫu thuật triệt để tứ chứng Fallot tại Bệnh viện Tim Hà Nội”, Luận án tiến sĩ y học, Hà Nội.

2. Lê Quang Thứu, (2009), "Kỹ thuật tạo hình van động mạch phổi một lá trong phẫu thuật sửa chữa hoàn toàn bệnh tứ chứng Fallot", Tạp chí Y hoc thực hành, $(690+691)$, tr. $38-43$.

3. Ngô Bảo Khoa, (2011), "Đánh giá kết quả trung hạn phẫu thuật sửa chữa tứ chứng Fallot có tạo hình van động mạch phổi một lá", Tạp chí Y học thục hành, (KHÔNG THÁY SÓ MÁY, TRANG MÂYY, CÓ Gì ANH TİM VÀ BỔ SUNG GIÙM EM).

4. Bove E. L., Hirsch J. C., (2006), "Tetralogy of Fallot", Surgery for Congenital Heart Defects, John Wiley \& Sons, $3^{\text {th }}$ Edition, (29), pp. $399-410$.

5. Dyamenahalli U, Mc Crindle BW et al, (2000), "Infuence of Perioperative Factors on Outcomes in Children Younger Than 18 Months After Repair of Tetralogy of Fallot", Ann Thorac Surg, (69), pp.1236-42
6. Jonas R. A., (2009), "Early Primary Repair of Tetralogy of Fallot", Semin Thorac Cardiovasc Surg Pediatr Card Surg Ann, (12), pp. $39-47$.

7. Kaushal S. K., Radhakrishanan S., Dagar K. S., Iyer P. U., Girotra S., Shrivastava S., Iyer K. S., (1999), "Significant intraoperative right ventricular outflow gradients after repair for tetralogy of Fallot: to revise or not to revise", Ann Thorac Surg, 68, pp. 1705 - 1713.

8. Kirklin J. W., Barratt-Boyes B. G., (2003), "Ventricular Septal Defect with Pulmonary Stenosis or Atresia", Cardiac Surgery, Churchill Livingstone, 3nd Edition, Volume 1, (24), pp. 946 - 1073.

9. Mark W et al. (2002), "PTFE Monocusp Valve Reconstruction of the Right Ventricular Outflow Tract", The Society of Thoracic Surgeons 20

10. Pozzi M., Quarti A., Corno A. F., (2006), "Tetralogy of Fallot", European Association for Cardio-thoracic Surgery.

11. Stewart R. D., Backer C. L., Young L., Mavroudis C., (2005), "Tetralogy of Fallot: Results of a Pulmonary Valve-Sparing Strategy", Ann Thorac Surg, (80), pp. 1431-1439.

12. Sasson $L$ et al (2013), "Right ventricular outflow tract strategies for repair of tetralogy of Fallot: effect of monocusp valve reconstruction", Eur J Cardiothorac Surg. 2013 Apr;43(4)

13. Turrentine M. W., McCarthy R. P., Vijay P., Fiore A. C., Brown J. W., (2002), "Polytetrafluoroethylene Monocusp Valve Technique for Right Ventricular Outflow Tract Reconstruction", Ann Thorac Surg, (74), pp. 2202 - 2205.

14. Van der Wall E. E., Mulder B. J. M., (2005), "Pulmonary valve replacement in patients with tetralogy of Fallot and pulmonary regurgitation: early surgery similar to optimal timing of surgery?", European Heart Journal, (26), pp. $2614-2615$. 